\title{
On the Connection Between Galaxies with Ultraviolet Excess
}

\author{
A. A. Yeghiazaryan \\ NAS RA V. Ambartsumian Byurakan Astrophysical Observatory (BAO) \\ Byurakan 0213, Aragatzotn Province, Armenia \\ E-mail: anahit@bao.sci.am
}

\begin{abstract}
A possible correlation between the characteristic parameters of physically coupled galaxies with ultraviolet excess is discussed. It is known that for mutually independent combinations the observed number of galactic pairs with Markarian components is greater than expected. It is shown, that the most of nearby Kazarian galaxies with ultraviolet excess form physical systems.
\end{abstract}

Keywords: galaxies - ultraviolet excess - physical connection - spectra.

The observational data indicate that characteristics of this galaxy largely depend on the characteristics of the surrounding galaxies, that is to say the correlation exists between characteristic of the shown galaxy and the characteristics of the members of the system in which this galaxy is included (Smirnov 1980, Giuricin 1985, Mahtesyan 1985). In the groups with relatively high density the galaxies are redder in the systems with relatively high number of elliptical galaxies, and elliptical galaxies are bluer in the systems where increased quantity of spiral galaxies is observed. There are also differences observed between isolated galaxies and galaxies that are members of multiple systems of galaxies. There are more emission galaxies in the galactic pairs and groups, and normal galaxies dominate among the isolated galaxies (Shuder 1981, Markarian 1882) whereas majority of galaxies belonging to pairs are peculiar (Sahakian 1975, Stoke 1978). There is an observed excess of galaxies with ultraviolet excess among the components of the galactic pairs, and with mutually independent combinations the observed number of pairs with Markarian components is distinctly larger than expected (Karachentsev 1981; 1984).

The above-mentioned suggests a possibility of correlation between characteristic parameters of physically coupled galaxies with ultraviolet excess. 
The subject of a possible physical link between nearby galaxies with ultraviolet excess from the Kazarian list was considered (Kazarian 1979a,b, 1980, 1982). Taking into consideration only those pairs, which simultaneously are galaxies from the Kazarian list, the distribution of the angular distances on the celestial sphere of the first three near-neighbors for all Kazarian galaxies were considered. The observed and the Poisson distributions of the first, second and third near-neighbors of the Kazarian galaxies did not coincide. It was seen from the distribution that in $25 \%$ of the galaxies the angular distances of the first near-neighbors are less than $10^{\prime}$. It was assumed that the majority of nearby galaxies with the ultraviolet excess from the Kazarian lists with angular distance less than $10^{\prime}$ compose physically related systems (Yeghiazaryan 1987). The first research data of the spectroscopic study of the first near-neighbors for 27 Kazarian galaxies demonstrated that 12 out of 14 binary systems which angular distances between components are less than $10^{\prime}$ form the physically related systems, which is consistent with the expected results (Kazarian 1987; Yeghiazaryan 1986a; 1986b; Yeghiazaryan \& Khachikian 1988a; 1988b).

The catalog of Kazarian galaxies with ultraviolet excess, published in 2010, consists of 706 galaxies, of which the values of redshifts for 502, and the activity classes for 150 galaxies are given (Kazarian et al. 2010). Among them there are 28 pairs and 2 triplets of physically connected galaxies. Further studies of the Kazarian galaxies have shown that notable groups of the Kazarian galaxies are indeed observed (Yeghiazaryan 1995; 2016; 2018).

Figure 1 shows the spectrogram of the galaxy Kaz 195, and the figure 2 shows the spectrogram of the galaxy Kaz 197, obtained in 2017 with the 2.6m telescope of the Byurakan Astrophysical Observatory.

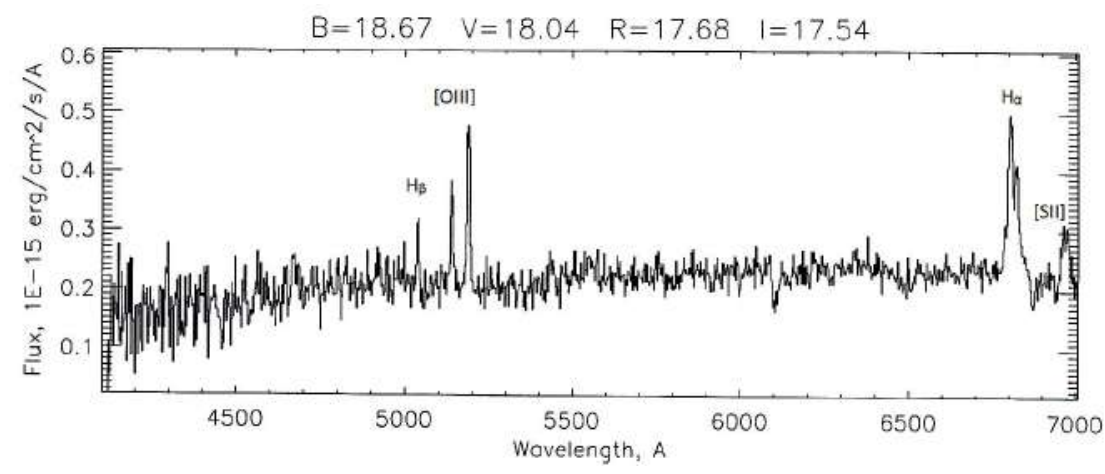

Figure 1: Spectrum of the galaxy Kaz 195 


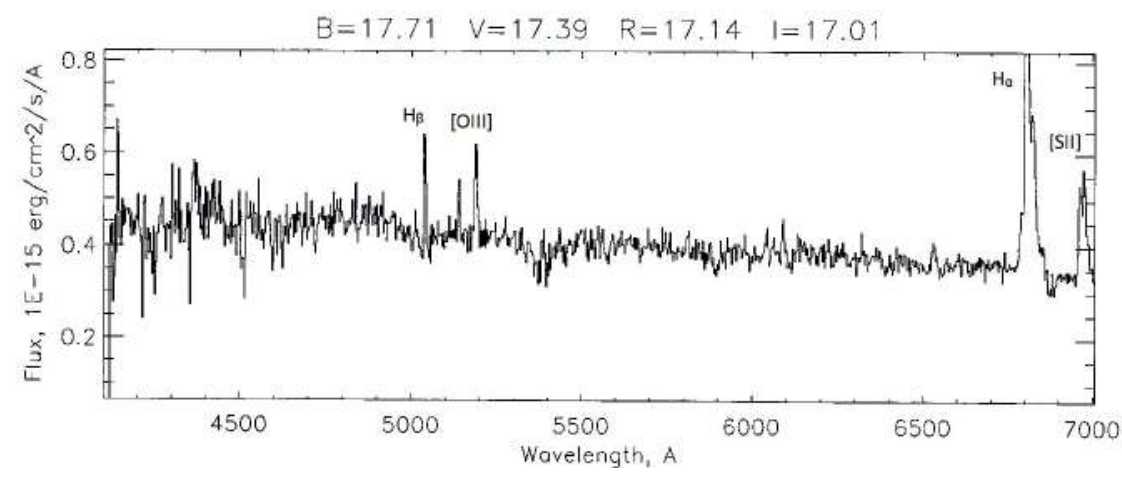

Figure 2: Spectrum of the galaxy Kaz 197

\begin{tabular}{|c|c|c|c|c|c|c|}
\hline Kaz & $\alpha$ & $\delta$ & SMC & m & $\mathbf{R}$ & V \\
\hline 195 & $18^{h} 14^{m} 01^{m}$ & $70^{\circ} 18^{\prime} 05^{\prime \prime}$ & $\mathrm{s} 3$ & 17.1 & 146.4 & 10980 \\
\hline 197 & $18^{h} 14^{m} 07^{m}$ & $70^{\circ} 17^{\prime} 38^{\prime \prime}$ & $\mathrm{s} 2$ & 17.2 & 146.1 & 10950 \\
\hline
\end{tabular}

Table 1: Parameters of galaxies Kaz 195 and Kaz 197

Table 1 shows the parameters of galaxies Kaz 195 and Kaz 197 (Kaz number, equatorial coordinates $\alpha$ and $\delta$, activity class, apparent isophotal magnitude, distance in Mpc, radial velocity in $\mathrm{km} / \mathrm{sec}$ ).

If classified, the studied galaxies with UV excess by their spectral features, among them there are many double systems with similar spectral characteristics of their components. Conducting a comprehensive study of closely spaced galaxies with ultraviolet excess would be beneficial in order to find out the correlation between individual physical characteristics and morphological features of the components of physically connected active galaxies.

\section{References}

Giuricin G.; Mardirossian F.; Mezzetti M.; Astron. Astrophys. Suppl., $1985,62,157$

Karachentsev I. D.; Karachensteva V. E.; AZh, 1974, 51, 724

Karachentsev I. D.; PAZh, 1981, 7, 3

Kazarian M. A.; Astrofizika, 1979a, 15, 5

Kazarian M. A.; Astrofizika, 1979b, 15, 193

Kazarian M. A.; Adibekyan V. Zh.; McLean B.; Allen R. J.; Petrosian A. R.; Astrofizika, 2010, 53, 1

Kazarian M. A.; Kazarian E.S.; Astrofizika, 1980, 16, 17

Kazarian M. A.; Kazarian E.S.; Astrofizika, 1982, 18, 512 
Kazarian M. A.; Kazarian E.S.; Astrofizika, 1983, 19, 213

Kazarian M. A.; Khachikian E. Ye.; Yeghiazaryan A. A.; Astrophys.

Space Sci.; 1982, 82, 105

Mahtesyan A. P.; So. Byu. 1985, 57, 13

Markarian B. E.; Astrofizika, 1972, 8, 165

Sahakian K. A.; Khachikian E. Ye.; Astrofizika, 1975, 11, 207

Shuder S. H.; Osterbroch D. E.; Astrophys. J., 1981, 250, 55

Smirnov M. A.; Comberg B. V.; Astrofizika, 1980, 16, 431

Stoke J. T.; Astron. J., 1978, 83, 348

Yeghiazaryan A. A.; So. Byu. 1986a, 58, 68

Yeghiazaryan A. A.; Astrofizika, 1986b, 25, 425

Yeghiazaryan A. A.; Astrofizika, 1995, 38, 690, 1995

Yeghiazaryan A. A.; Astrofizika, 2018, 61, 455

Yeghiazaryan A. A.; Khachikian E. Ye.; So. Byu. 1988a, 60, 1

Yeghiazaryan A. A.; Khachikian E. Ye.; So. Byu.; 1988b, 60,13

Yeghiazaryan A. A.; Nazaryan T. A.; Hakobyan A. A.; J. Astrophys. Ast., 2016, 37, 1

Yeghiazaryan A. A.; Zaratsian S. V.; Mahtesian A. P.; So. Byu.; 1987, 59,53 